\title{
VISUAL EDUCATION ON CONSTRUCTION SITES USING BIM
}

\author{
SEBASTIAN SEIß \& BILAL SHAH \\ Bauhaus-University Weimar Chair Construction Management, Germany
}

\begin{abstract}
The advancement in building information modelling (BIM) technology has largely benefited architecture, engineering, and construction (AEC) stakeholders. Automation and visualization offered by different BIM tools are regularly used by architects, engineers, and planners to make the planning and design process more efficient and accurate. However, the potential of BIM for helping construction site workers is not fully realized; the existing methods for using intelligent 3D BIM models to instruct site workers are limited and can potentially develop. A BIM-based 4D visualization is a practical use case for showing the sequence of site tasks to workers and enables them to identify their scheduled tasks. By now, 4D BIM does not support workers in the process of site implementation and work safety. The use of videos for instruction purposes has been an effective practice in other sectors, but it has not been used in AEC for facilitating site works. Thus, the research develops a method by which video tutorials are connected to task objects in a $4 \mathrm{D}$ model. The aim is to access instructional videos following the scheduled tasks in the 4D model to construction workers. The proposed method allows site workers to visualize a sequence of site tasks on a 4D model and to understand construction tasks by an automized displaying of linked instruction videos. The procedure is evaluated on a case study and a survey of workers and site managers. Based on the analysis of survey results, factors such as future relevance, practicality, and usability of the proposed method are clarified, and future works are recommended.
\end{abstract}

Keywords: visual education, BIM, 4D planning, health and safety, construction operations.

\section{INTRODUCTION}

In a construction project, transferring information about the intended product to the lowest level is of utmost importance. The on-site workers do not understand the technical terms, which are generally used on construction sites by engineers and site managers. It is tough to communicate to site workers about performing a particular construction task with expected accuracy. The communication barriers, e.g., language and the inability of site workers to understand verbal instruction due to the complexity of the task, have been a long-stayed problem in the construction industry. This communication problem results in faulty and incomplete work. Due to inadequate advancement in exploring ways to instruct low-skilled workers, there has been a heavy reliance on verbal instructional methods on site.

The advancements in the method of BIM have allowed us to explore visualization in the construction industry in the most productive way. The progress in BIM has benefited the stakeholders involved at the highest level, but it has not been used to help the site workers significantly. The data-enriched 3D BIM models can be used as an instructional tool for all levels, i.e., Engineers, Architects, site workers, trainees, and owners. The intelligent 3D Models resulting from the BIM process allow us to link extra information with the model objects as a kind of information repository. The information can be accessed throughout the lifecycle of the building for different purposes. The interoperability property of the objectoriented models enables the exchange of project information among participants.

The use of videos and visualization for instructional and safety purposes has been a massive success in different sectors. One of the main reasons for videos being an effective medium of instruction is the self-learning ability. Also, instructional video clips help to 
build confidence for executing a particular task by visualizing each detail of its execution process. The development of such a visual instructional model for low-skilled workers can prove to be an effective tool in communicating construction tasks.

The current usage of construction videos for instructional tasks will be explored before the concept development. The research focused on an automatized linking of instruction videos to the 3D Model objects and the 4D schedule of a BIM. The studies proved to be an important development in visual education for site workers and paved the way for this research. It is vital for avoiding miscommunication to develop an approach that focuses on construction activities and instruction for the steps involved in site and work safety tasks. The use of 4D BIM provides an effective tool to develop a visual environment in which instructional video tutorials can be linked to the scheduled tasks of the 4D model. In this way, site workers can visualize the sequence of construction activities through 4D simulation and get the necessary work instructions to execute the tasks. This study aims to develop a method for linking instructional and safety videos with the 4D model to guide site workers for their scheduled tasks and educate them about the sequence of activities by 4D simulation.

\section{BACKGROUND ON VISUAL EDUCATION}

Visualization is defined as the learning tool used to convey, portray, educate and store data in all practical fields, from architecture to chemistry, meteorology to radiology, and finance to history. If the visualization is planned and designed appropriately, it can enhance human information processing [1].

Multimedia-based learning is certainly more effective than typical verbal instructions, as it provides an interactive, self-paced learning environment and appeals to different learning types. The study of Issa states, "Multimedia presentation enhances people's ability to visualize; it is the impetus to learning that takes instruction from the highly subjective means of verbal communication and develops mental images that help many people understand new information more clearly." Additionally, this learning method helps develop confidence in learners; it provides the opportunity to learn and improve themselves instead of being trained, which increases worker's interest in getting more involved in tasks [2].

Many researchers studied visual education of different forms to educate site workers with difficulties, e.g., language barriers, low experience, and low skill in understanding verbal instructions. Visual education by videos or pictures has also been effective for instructing the assembly and installation of various home appliances. Some of the examples of the use of visual education as an effective medium in different research are discussed as follow. The differences between assembly and construction task instructions were identified by Mourgues and Fischer [3]. These differences influence the attributes gained from the above-stated principles. The main differences are the user knowledge about the task, operation environment, and complexity of tasks. The study identified problems with the existing verbal instructional method on the construction site and defined a template with the work structure and works instructions for cast-in-place concrete labourers. The template used can be observed in Fig. 1.

The template was validated in the field and found to be effective compared to the traditional verbal method. Labourers and site personal preferred the instruction based on this template as it reduced rework and questions. The template contributed significantly to the cast-in-place construction domain and provided a strong foundation for developing new methods for visual site instruction [3]. 


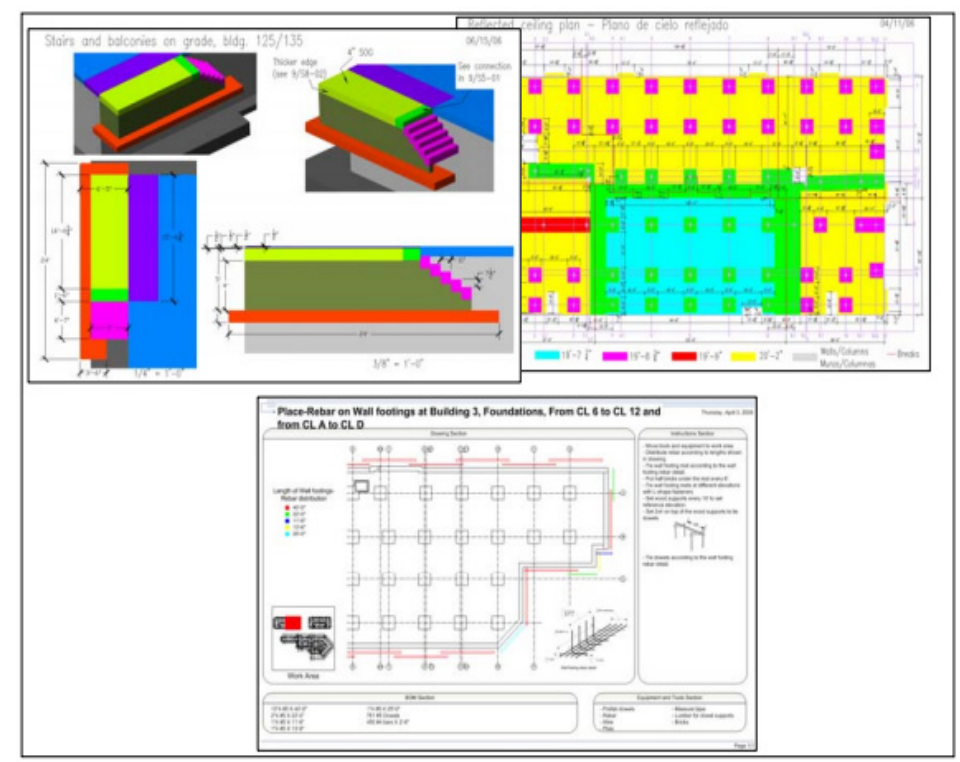

Figure 1: The template used for cast-in-place concrete labourers [3].

One of the advantages of using 3D visualization is the training of workers with limited education. As most site workers might be less educated, it is difficult for them to follow written instructions. The other problem could be language, meaning construction workers from different regions find it difficult to follow complex tasks, as they are unfamiliar with the local language. 3D visualization is an effective training tool that can address these problems and communicate tasks to workers better.

To confirm the usability of $3 \mathrm{D}$ visualization, a training module was modelled by Clevenger and Del Puerto, which focused on the relationship of assembling construction elements such as walls and safety provisions. The wall assembly model was initially developed to identify issues during the design phase and prevent them from becoming a problem. Once the model was prepared, it was realized that it could serve additional purposes and could be used as content for a training module. (see Fig. 2). The safety provisions recommended by Occupational Safety and Health Administration (OSHA) is associated with every construction stage [4].

Video tutorials are used for on-site safety instructions in animated videos to create awareness in site workers regarding site safety. The United States Department of Labourer covers different construction features through video tutorials. It creates awareness among workers about different unwanted situations during the execution process of various tasks [5].

The verbal instructional method used on site is observed to have limitations in instructing site workers because of their limited knowledge and language barriers. The study introduces a video-based instructional approach in which video with systematic instructions for a construction task is considered as an alternative instructional method. The use of video tutorials encourages self-learning and ensures efficient transfer of knowledge. Video tutorials have played an important role in assisting users in learning software tools 


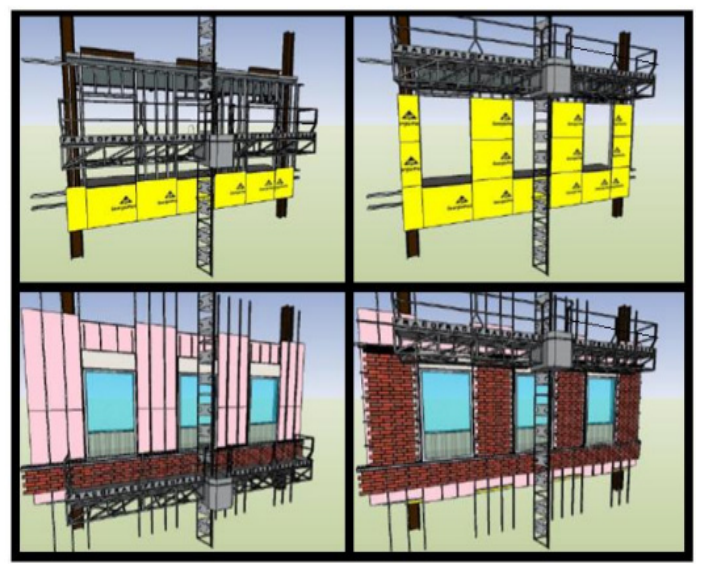

Figure 2: 3D-visualization of a wall describing construction sequence and safety controls [4].

related to the construction industry and creating site safety awareness. A general approach for developing task-related videos is presented by Nasir and Bargstädt, which comprises four phases; needs recognition, content design, making instructional content, and assessment. BIM 3D models are the primary source for creating a virtual environment of a video tutorial; therefore, videos developed by this approach are called BIM-based video tutorials for construction tasks. It is expected that videos developed through this process would help simplify the construction tasks for site workers [6], [7].

\section{METHODOLOGY}

To implement the idea of providing scheduled-based instructional content to the site worker, combining the $3 \mathrm{D}$ model with a schedule to execute activities should be helpful. The combination of the 3D model with time information is referred to as a 4D model. The other main aspect of this research is discovering how external instructional content such as videos related to scheduled tasks and safety are connected to 4D-model information. In this way, the proposed method would help site workers understand the sequence of tasks and methods to perform the scheduled task with the required safety.

The research approach carried out for this study is design-based, proposed by Verschuren and Hartog [8]. The stages mainly comprised the following:

1. Needs identification and making assumptions for the proposed model

2. Development of a concept in a way that satisfies the needs

3. Creation and implementation of a test version

4. Evaluation

This study focuses on creating an alternative instruction method by identifying its requirements and defining assumptions for the proposed new method. The requirements are based on a literature review, and an iterative coordination process with Hochtief end users. Based on assumptions and requirements, the concept is developed, after which a test version of the proposed concept is created and implemented on the target users. The test version embodies the characteristics of the expected final product. Finally, it is evaluated by the end-users to find out if it satisfies the requirements and assumptions. The validation is 
done by a 4D model combined with an instructional video on target users. For measuring the satisfaction level, staff working at different positions are interviewed. The results are structured using a standardized questionnaire and analyzed to identify the problem stage.

\section{SYSTEM DEVELOPMENT FOR VISUAL EDUCATION USING BIM}

The focus of the study is to provide schedule-based instructional content; therefore, DesiteMD is used. DesiteMD enables the connection of 3D model objects to time information like Navisworks and provides the user with a full Application Programming Interface (API) access. Once 3D model objects are connected to the activity schedule, the model is referred to as the 4D model, which shows construction sequencing of different activities through 4D simulation. The other part of the study is the connection of instructional and safety videos to 4D model information. It is achieved by using the API of DesiteMD; the API offers functions that link instruction videos with a 4D model and automatically display the videos to the 4D schedule sequence.

As the target user of the system is a site worker, the system should allow easy access to the intended content. The vital aspect of this study is to develop a simple user interface. The webform module in DesiteMD API receives signals from the UI (user interface) of DesiteMD; it establishes relationships with scheduled tasks for this study [9].

The concept of the system is illustrated in Fig. 3. The system is based on a two-layer architecture of user interface on the top and the software system on the bottom. The visual education concept is based on a 4D BIM and links videos to the provided tasks in the model. First, the user will run the 4D sequence of the model by the user interface. During the animation, DesiteMD searches for links between the building object, schedule tasks, and video links. The database contains the videos as links based on Uniform Resource Locator (URL) of common online video platforms in relation to standardized tasks. If the model contains an object and the object is related to one or multiple tasks of a schedule, the program will reason the related video link. The video will be then automatically displayed in the user interface. The videos are implemented by iFrame in DesiteMD and can be provided by each video platform like YouTube or Vimeo. It becomes clear that the tasks used in the schedule have to be according to the task stored in the database to ensure a link between the task and the provided video.

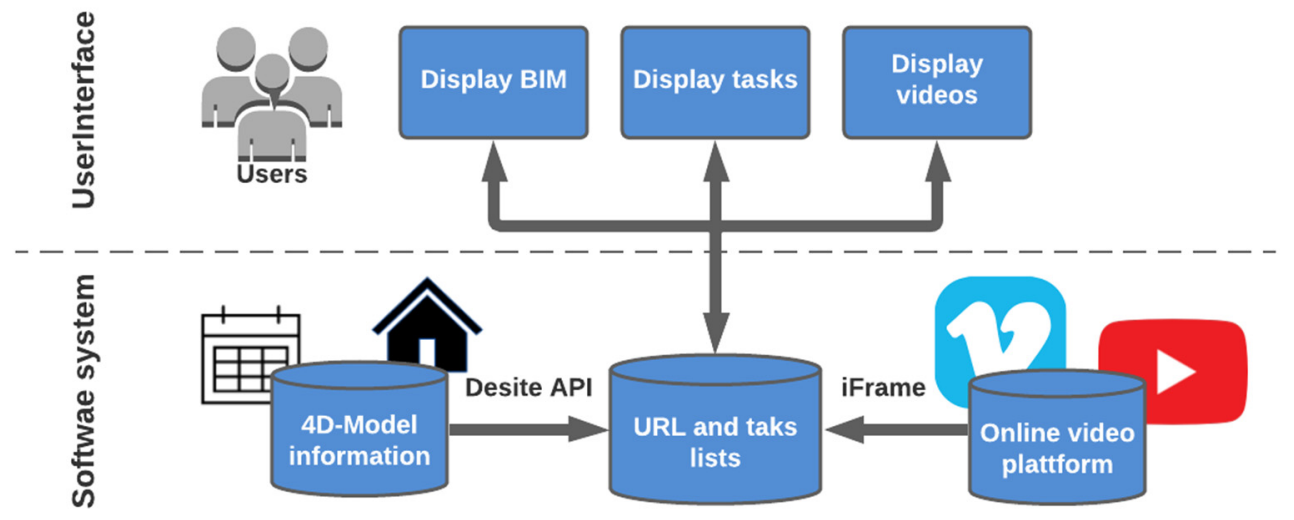

Figure 3: Concept for assigning videos to 4D model information. 


\subsection{User interface and access to instructional videos}

An overview of the user interface is given in Fig. 4. On the top of the software application is the usual menu of the software. The Screen is divided into two main areas. On the left side, information about the schedule and the videos according to the task of the 4D model are illustrated. On the right side, the BIM is visualized according to the colour related tasks of the schedule. In this example, the concreting process of a column is visualized in the video window on the left side, including the scheduled task with a start, end date and duration. The column to be concrete is visualized in the centre of the 4D-BIM in yellow.

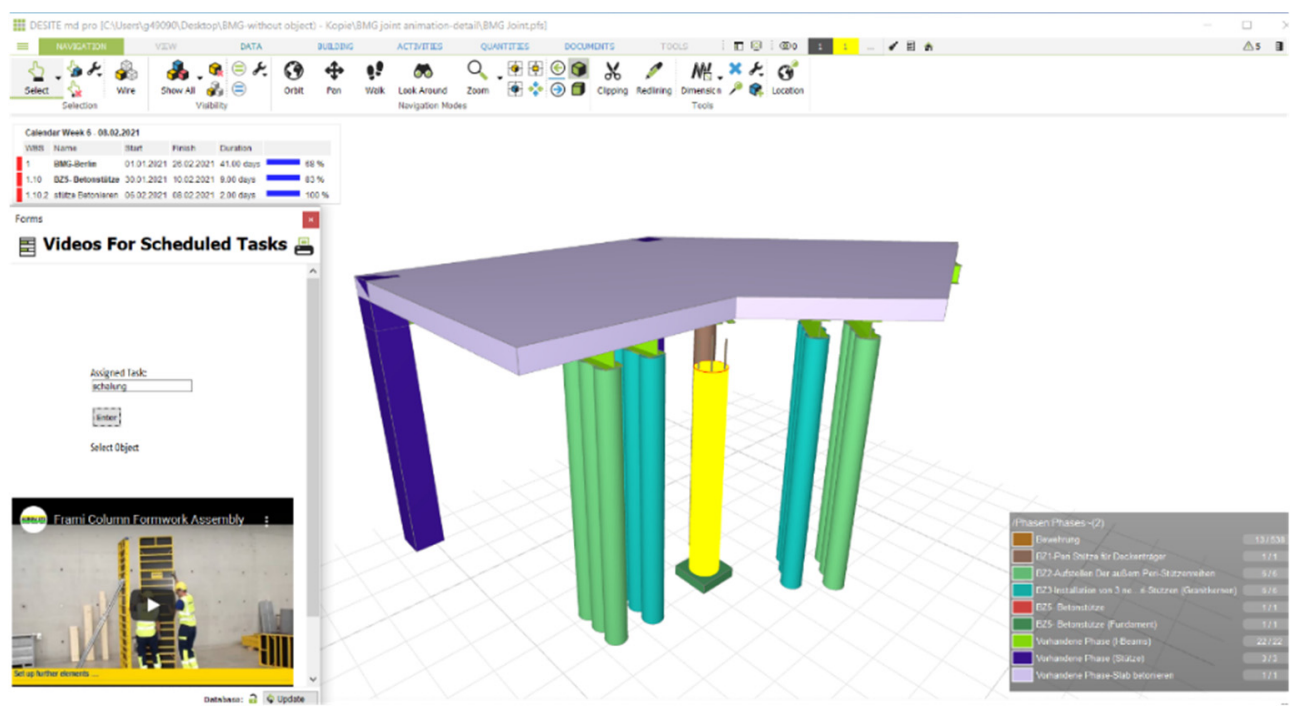

Figure 4: The user interface of DesiteMD.

The user can individualize the user interface to add more information according to the task needs, like concrete class or used formwork. The software DesiteMD enables the construction workers to watch videos on multiple mobile devices accessible to site supervisors and construction workers.

\subsection{Automatic access of instructional videos based on a 4D BIM for visual education on site}

As discussed earlier, the system can access all the videos related to the $4 \mathrm{D}$ object. It is used for watching videos that display the start to finish process of constructing an object, e.g. if the column is selected, it will display videos related to reinforcement, formwork and concreting. The displayed videos depend on the represented activity of the schedule and the selected object by the user. For example, if the user selects an identified 3D object representing activity, e.g. column formwork, and all task videos related to the column formwork will appear. The user will select the appropriate video, which resonates with the scheduled task to watch it (see Fig. 5). 


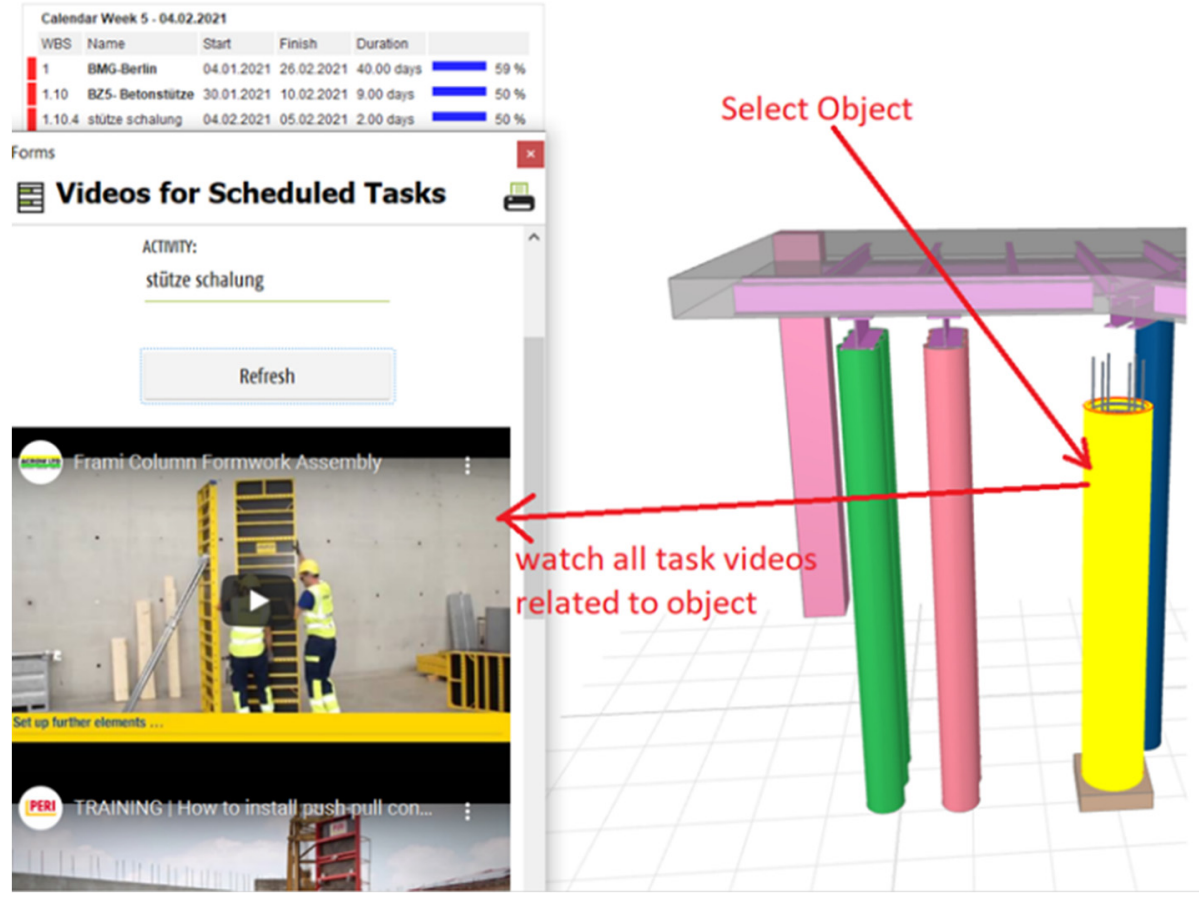

Figure 5: Access all task video connected to 4D activity and the building object.

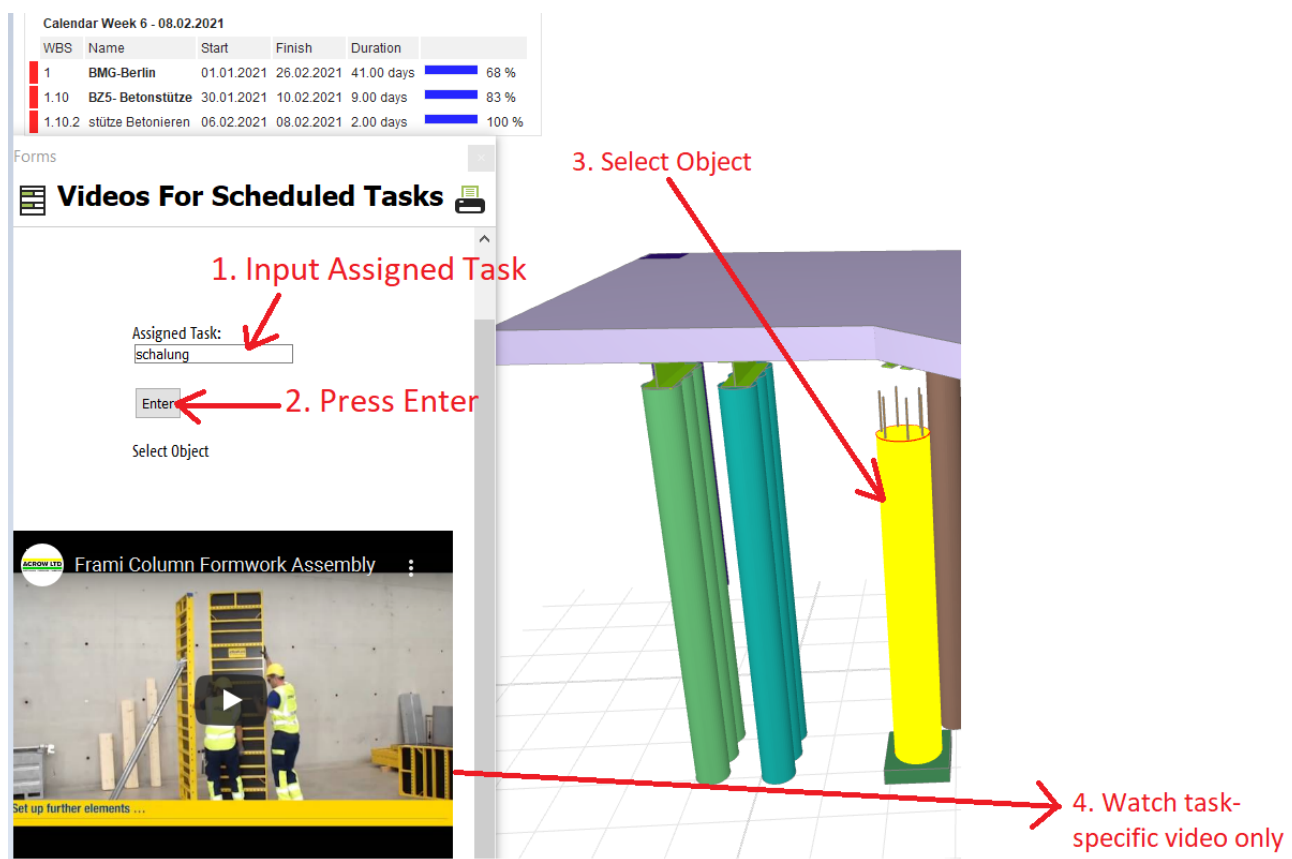

Figure 6: Process for watching task-specific video through 4D-model. 
To access only task-specific videos from objects connected to multiple activities, additional control called "Assigned Task" is added to the user interface of the web service module.

It should be noted that the colour scheme is used for identifying a task-specific object in the same way as used for displaying all videos linked with task objects. The selection of objects follows the same process as mentioned in Fig. 6. Apart from showing videos for activities not connected to objects, the other advantage of using this method is that it enables construction workers to watch only videos assigned, especially their scheduled activity. The selected column object in Fig. 7 is connected to more than one activity, but due to user input "Formwork" in the field "Assigned Task," only formwork video appears.

\subsection{Warning for activities with special safety instructions}

The use of 4D simulation can highlight activities involved in different stages that require special safety instructions. After viewing the 4D simulation, access to safety video follows the procedure defined in the previous section. Fig. 7 shows the appearance of a safety warning for current activity in 4D animation. The object of the current activity is highlighted with red colour by default. The 4D animation stops on the activity "Ring beam concreting," and a safety warning appears. The activity name and building object are noticed. By clicking the "OK" button, the 4D simulation will continue.

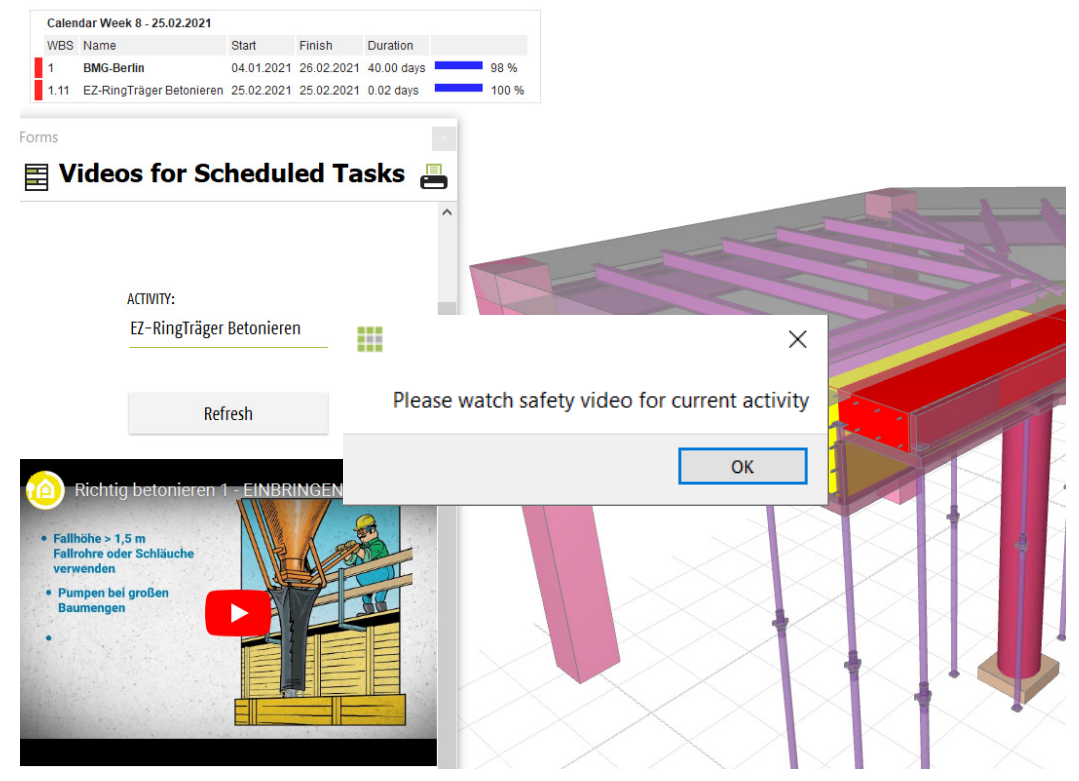

Figure 7: Safety video for activity "Ringbeam Concreting".

As a warning had appeared on activity "Beam Concreting" during 4D animation, task object is selected, resulting in displaying safety video related to concrete.

To watch the safety video in a 4D model that displays task-specific content, user input, e.g., "Safety instructions" in the field "Assigned Task," is required before selecting the object. 
Although 4D simulation is used to get aware of activities that require special safety precautions, its start/pause-control also facilitates a user to watch an instructional video related to the required task at any point. It provides a possibility to the user for viewing construction sequence and video content simultaneously.

\section{SURVEY ON THE PROPOSED SYSTEM}

The questionnaire was sent to 110 employees of the construction company Hochtief. Out of 110 persons, 39 had participated. The questionnaire contained the link for video, which displayed 4D animation and methods for accessing instructional videos through 4D model related to different construction activities. The video was edited so it explained each step by using text and mock characters.

The survey investigated that $87 \%$ of the participants believed that videos related to scheduled tasks with the BIM model could help site workers execute tasks effectively. Especially benefits in the visualization "1 picture is worth 1000 words!" and the missing need for language skills are highly positive rated by the participants.

The participants realize the potential for using such 4D animation and video connection for solving problems. One of the significant aspects noticed in the answers is the emphasis on the need for visual-based instructions compared to verbal or written. It is clear from the answers that communication problems greatly influence the level of understanding for complex tasks; therefore, using such a visual environment can help in better interpretation of tasks. The answers reflect on the importance of using proper up-to-date videos related to more complex tasks. It is essential to provide access and a clear method to site workers to use such $4 \mathrm{D}$ models and watch video material through it.

Furthermore, $65 \%$ of the participants thought that the proposed instructional method is relevant and usable in the future; around $35 \%$ of the participants expressed either doubt or considered it irrelevant. Therefore, the process is deemed suitable for future tasks because it can make challenging tasks easily understandable; if the proposed method is used to its full potential, it can limit human error by bridging the communication gap.

It is concluded from the recommendations that the involved site workers, i.e., site managers, labourers need to be educated regarding the potential benefits of this method and the procedure to use it. Implementing a practical task can be a useful practice because the proposed method will only convince site workers if they see the practical outcome of the method to understand the task in a better way. The potential users of this method are site workers; therefore, the 4D model should be accessible to them, and the method to access content should be simple and clearly explained. The recommendations also reflected the possible use of instructional mediums other than videos, e.g., detailed drawings, technical data, etc. The connection of instructional videos with 4D model objects is considered a useful practice. Still, the videos should focus on more complex site tasks rather than simple formwork, as shown in the survey video.

\section{CONCLUSION AND FUTURE WORK}

The proposed system enables construction workers and site managers to access the right instructional and safety video content at the right time by linking video content through an algorithm and database. The participants, especially the site manager, realize the potential to use such 4D animation and video connection to solve daily construction site problems. One of the major aspects noticed in the answers is the need for visual-based instructions compared to verbal or written. It is clear from the answers that communication problems greatly influence the level of understanding for complex tasks; therefore, using such a visual environment can help in better interpretation of tasks. It can also be derived that the 
approach is used for increased coordination among planners and contractors regarding challenging tasks, e.g., heavy reinforcement. The animation displaying a correct work sequence, e.g., reinforcement, can help less experienced engineers implement the reinforcement plan correctly. The answers reflect on the importance of using proper up-todate videos related to more complex tasks. It is essential to provide access and a clear method to site workers to use such 4D models and watch video material through it.

It is concluded that the involved site workers, i.e., site managers, labourers need to be educated regarding the potential benefits of this method and the procedure to use it. Therefore, the 4D model and videos should be accessible by mobile devices. The recommendations also reflect the possible use of instructional mediums other than videos, e.g., detailed drawings, technical data, etc. The connection of instructional videos with 4D model objects is considered a helpful practice. Further, the development of a video library focusing on more challenging site tasks is necessary. The contained videos can therefore be real or animated. As videos are assigned to the activities based on their name, it is essential to create a BIM standard for naming construction activities. In this way, the method will be generalized and usable for all the companies working with BIM. In further research, the script should be enveloped to add a rating option to the user interface so that workers can value the instructional video per its helpfulness.

\section{ACKNOWLEDGEMENT}

We want to thank the company Hochtief for their support in processing this work and participating in the survey.

\section{REFERENCES}

[1] Tversky, B., Spatial Schemas in Depictions, 2001.

[2] Issa, R.R.A., Cox, R.F. \& Killingsworth, C.F., Impact of multimedia-based instruction on learning and retention. Journal of Computing in Civil Engineering, 13(4), pp. 281290, 1999.

[3] Mourgues, C. \& Fischer, M., A work instruction template for cast-in-place concrete construction laborers. Center for Integrated Facility Engineering, Stanford University. Working Paper 109, 2008.

[4] Clevenger, C. \& Del Puerto, C.L., Using 3D visualization to train Hispanic construction workers. Associated Schools of Construction 2010, 2011.

[5] Nasir, A.R., A Digital Task Instruction Model for Low-skilled Construction Workforce, Bauhaus-Universitätsverlag Weimar im Jonas Verlag für Kunst und Literatur GmbH, 2017.

[6] Nasir, A.R. \& Bargstädt, H.J., An approach to develop video tutorials for construction tasks. Procedia Engineering, 196, pp. 1088-1097, 2017.

[7] Bargstädt, H., Nasir, A.R. \& Ignatova, E., Can BIM support better working conditions for low-skilled labor. CONVR 2014, p. 44, 2014.

[8] Verschuren, P. \& Hartog, R., Evaluation in design-oriented research. Quality and Quantity: International Journal of Methodology, 39(2005) pp. 6-39, 2005. DOI: 10.1007/s11135-005-3150-6.

[9] DesiteMD-API, DesiteMD (API). API (Tutorial). 